\title{
Aortic Stenosis Patients With Transcatheter Aortic Valve Replacement: Caution Recommended With Renal Failure During Hospitalization
}

\author{
Pawandeep Kaur ${ }^{1}$, Temitope Ajibawo ${ }^{2}$, Timiiye Yomi ${ }^{3}$, Neev Patel ${ }^{4}$, Mizba Baksh ${ }^{5}$, Edmond Okotcha ${ }^{6}$, \\ Saurabh Kataria ${ }^{7}$, Rikinkumar S. Patel ${ }^{8}$ \\ 1. Medicine, Sri Guru Ram Das Institute of Medical Sciences and Research, Amritsar, IND 2. Internal Medicine, \\ Brookdale University Hospital Medical Center, New York City, USA 3. Medicine, University of Benin School of Medicine, \\ Benin City, NGA 4. Medicine, Byramjee Jeejeebhoy Medical College, Ahmedabad, IND 5. Internal Medicine, Dr. \\ Nandamuri Taraka Rama Rao University of Health Sciences, Vijayawada, IND 6. Medicine, Vinnytsia Pirogov National \\ Medical University, Vinnytsia Oblast, UKR 7. Neurology, University of Missouri, Columbia, USA 8. Psychiatry, Griffin \\ Memorial Hospital, Norman, USA
}

Corresponding author: Rikinkumar S. Patel, rpatel_09@arcadia.edu

\section{Abstract \\ Objective}

Our study aimed to assess the risk of in-patient mortality due to renal failure and other comorbidities in aortic stenosis (AS) patients undergoing transcatheter aortic valve replacement (TAVR).

\section{Methods}

We conducted a cross-sectional study using a Nationwide Inpatient Sample (NIS, January 2010 to December 2014) from the United States and included 33,325 patients with a primary diagnosis of AS. Logistic regression was used to evaluate the odds ratio (OR) for in-hospital mortality in AS by comorbidities including renal failure.

\section{Results}

The prevalence of renal failure in AS patients is $29.2 \%$, and a higher proportion were males (60.1\%) and nonwhite (14.1\%). Major loss of function (96.6\%) and in-hospital mortality (5.1\%) were also proportionally higher in prevalence. Female patients (OR 1.35, 95\% CI 1.20-1.51) had higher odds of in-patient mortality in AS patients. Race was a non-significant predictor for mortality risk. Patients with comorbid coagulopathy (OR 2.02, 95\% CI 1.79-2.27) and heart failure (OR 1.62, 95\% CI 1.39-1.89) have increased mortality in AS inpatients. After controlling confounders, renal failure was significantly associated with increased in-

Received 07/16/2020

Review began 07/16/2020 Review ended 07/17/2020 Published 07/25/2020

\section{(c) Copyright 2020}

Kaur et al. This is an open access article distributed under the terms of the Creative Commons Attribution License CC-BY 4.0., which permits unrestricted use, distribution, and reproduction in any medium, provided the original author and source are credited.

\section{Conclusion}

Renal failure was prevalent in AS patients and was an independent factor that increases the risk of inhospital mortality by $43 \%$. Due to worse outcomes, more studies are required to evaluate risk-benefit ratio and strategies to improve health-related quality of life in post-TAVR patients with renal failure, and optimally decrease inpatient mortality.

Categories: Cardiology, Internal Medicine, Nephrology

Keywords: renal failure, acute kidney injury, aortic stenosis, new generation tavr, tavr, mortality

\section{Introduction}

Aortic stenosis (AS) is a common valvular abnormality and is ranked as the third most common cardiovascular disease. In the general population, prevalence is around $0.4 \%$ and about $1.7 \%$ in the elderly population above 65 years of age [1]. A rise in prevalence is seen in AS with increasing age with about $4 \%$ of elderly aged above 85 years are affected by worsening aortic sclerosis and AS. Some of the factors associated with the progression of AS are older age, cigarette smoking, and comorbidities like hypertension, obesity, diabetes, dyslipidemia, chronic renal disease, atherosclerosis, and coronary artery disease (CAD). Other hemodynamic-related factors (left ventricular systolic dysfunction and/or low cardiac output), and aortic valve-related factors like a bicuspid aortic valve, degenerative AS, valve calcification and regurgitation also worsen the disease process [2].

Nearly 125,000 individuals in the United States began treatment for end-stage renal disease (ESRD) in 2016 and more than 726,000 (two in every 1,000 individuals) were on dialysis or were living with a kidney 
transplant. The incidence of ESRD is higher among men and African Americans (three times more likely than whites). The total expenditure on medicare beneficiaries with renal disease was about \$100 billion in 2015 which comprised over $\$ 64$ billion invested for all medicare beneficiaries with chronic kidney disease [3]. Most patients with ESRD have numerous comorbidities along with experiencing the effects of advanced AS. The prognosis of ESRD patients is affected by the presence of comorbidities like peripheral vascular disease, $\mathrm{CAD}$, congestive heart failure (CHF), diabetes, and chronic pulmonary disease with nearly $50 \%$ one-year survival in elderly patients on dialysis [4].

Preoperative severe renal disease and also acute kidney injury (AKI) are major predictors for one-year mortality in transcatheter aortic valve replacement (TAVR) patients. Post-TAVR individuals with AKI had a mortality rate of $7.8 \%$ to $29 \%$ and two to eight times higher risk compared to patients who did not have AKI $[5,6]$. Individuals with ESRD and AKI have increased in-hospital mortality, increased occurrence of inhospital complications, and poor outcomes following TAVR [7]. Individuals with advanced AS and ESRD undergoing TAVR have a remarkably elevated risk of mortality at 30 days and one-year and increased in-hospital mortality compared to non-dialysis patients [4].

In our study, we aim to discern the differences in demographics and comorbidities, and inpatient outcomes including the severity of illness, length of stay (LOS), total charges, and mortality between AS patients undergoing TAVR with versus without renal failure. Next is to evaluate the prevalence and risk of in-hospital mortality due to renal failure in post-TAVR patients.

\section{Materials And Methods}

\section{Data source}

A retrospective cross-sectional study was conducted using the National Inpatient Sample (NIS) database from January 2010 to December 2014. The NIS is the largest inpatient database from the healthcare cost and utilization project (HCUP) that includes patient records from about 4,411 hospitals and covers 45 states in the United States [8].

\section{Inclusion criteria}

We included adult inpatients $\geqslant 18$ years of age with a principal discharge diagnosis of AS $(N=33,325)$ by identifying the primary diagnostic field in the NIS for the International Classification of Diseases, ninth revision (ICD-9) diagnosis codes for 395.0, 395.2, 396.0, 396.2, 424.1 or 746.3, and principal procedure of TAVR (ICD-9 procedure codes 350.5 or 350.6 ). The study population was grouped by comorbid renal failure (RF) by identifying the co-diagnoses fields for ICD-9 codes of 403.01, 403.11, 403.91, 404.02, 404.03, 404.12, 404.13, 404.92, 404.93, 585.3, 585.4, 585.5, 585.6, 585.9, 586, v42.0, v45.1, v45.11, v45.12, v56.0-v56.32, or v56.8.

\section{Variables of interest}

Demographic variables assessed in this study included age, sex, and race. To measure the differences in inpatient outcomes in AS patients undergoing TAVR by comorbid renal failure, the following variables were included: severity of illness, LOS, total charges, and in-hospital mortality. The severity of illness in the NIS is stratified by minor, moderate, major, and extreme loss of body functions. The in-hospital mortality is the number of inpatient deaths and is all-cause. The LOS is calculated by the number of nights the patient was hospitalized for AS and the total charges during hospitalization excluded professional fees or other noncovered charges during HCUP data processing [9].

Comorbidities are coexisting conditions to AS, and based on existing literature, we identified comorbidities including CHF, chronic pulmonary disease, and coagulopathy using ICD-9 diagnosis codes [9].

\section{Statistical analyses}

We used descriptive statistics and cross-tabulation to discern the differences in demographics and hospital outcomes, and comorbidities between AS inpatients with versus without RF. Independent sample t-test was used to measure the differences in continuous variables: LOS and total charges. We applied the discharge weight provided in the NIS to get a national representation of the study population [9]. We used a binomial logistic regression model to evaluate the odds ratio (OR) for in-hospital mortality by comorbidities including comorbid RF in AS inpatients with TAVR, and the model was adjusted for and age, sex, and race. A P-value $<0.01$ was used to determine the statistical significance of all the statistical analyses that were conducted using the Statistical Package for the Social Sciences (SPSS), version 26 (IBM Corp., Armonk, NY).

\section{Ethical approval}

To protect the privacy of patients and treatment information, the related identifiers were de-identified during the HCUP NIS data processing. We were not required to obtain institutional review board permission, as we utilized the publicly available de-identified data in our study [8]. 


\section{Cureus}

\section{Results}

We analyzed a total sample of 33,325 patients with AS, and 29.2\% $(\mathrm{N}=9,740)$ had comorbid RF. A higher proportion of AS patients with comorbid RF were male (60.1\% vs. $47.5 \%)$ and non-white being black, Hispanic, or other (14.1\% vs. 9.7\%) compared to the non-RF cohort. Patients in the RF cohort had a higher prevalence of comorbidities compared to non-RF cohort ( $90.5 \%$ vs $60.2 \%$ ), with a higher proportion of CHF (12.7\% vs $11.2 \%$ ) and coagulopathy (27.4\% vs $21.3 \%$ ). However, no statistically significant difference was seen in the prevalence of chronic pulmonary diseases between both cohorts. Major loss of function or severity of illness was seen in a higher proportion of AS patients in the RF cohort ( $96.6 \%$ vs $92.5 \%$ ), and so was the In-hospital mortality (5.1\% vs 3.6\%) compared to the non-RF cohort. Statistically, there was a significant difference between the mean LOS and total charges as shown in Table 1.

\begin{tabular}{|c|c|c|c|c|c|}
\hline \multirow{2}{*}{ Variable } & \multicolumn{2}{|c|}{ Renal failure (-) } & \multicolumn{2}{|c|}{ Renal failure (+) } & \multirow{2}{*}{ P-value } \\
\hline & $\mathrm{N}$ & $\%$ & $\mathrm{~N}$ & $\%$ & \\
\hline Total inpatients & \multicolumn{2}{|l|}{23585} & \multicolumn{2}{|l|}{9740} & - \\
\hline Mean age (SD), in years & \multicolumn{2}{|c|}{$81.2(8.28)$} & \multicolumn{2}{|l|}{$81.7(7.55)$} & $<0.001$ \\
\hline \multicolumn{6}{|l|}{ Sex } \\
\hline Male & 10240 & 47.5 & 7085 & 60.1 & \multirow{2}{*}{$<0.001$} \\
\hline Female & 11300 & 52.5 & 4700 & 39.9 & \\
\hline \multicolumn{6}{|l|}{ Race } \\
\hline White & 18185 & 90.3 & 9285 & 85.9 & \multirow{4}{*}{$<0.001$} \\
\hline Black & 600 & 3.0 & 560 & 5.2 & \\
\hline Hispanic & 515 & 2.6 & 370 & 3.4 & \\
\hline Other & 835 & 4.1 & 595 & 5.5 & \\
\hline \multicolumn{6}{|l|}{ Comorbidities } \\
\hline Chronic pulmonary disease & 7190 & 33.4 & 4090 & 34.7 & 0.015 \\
\hline Congestive heart failure & 2410 & 11.2 & 1500 & 12.7 & $<0.001$ \\
\hline Coagulopathy & 4590 & 21.3 & 3230 & 27.4 & $<0.001$ \\
\hline \multicolumn{6}{|l|}{ Severity of illness } \\
\hline Minor/moderate loss of function & 1605 & 7.5 & 400 & 3.4 & \multirow{2}{*}{$<0.001$} \\
\hline Major loss of function & 19935 & 92.5 & 11385 & 96.6 & \\
\hline \multicolumn{6}{|l|}{ Other hospital outcomes } \\
\hline Mean length of stay (SD), in days & \multicolumn{2}{|l|}{$7.1(6.14)$} & \multicolumn{2}{|l|}{$8.7(7.54)$} & $<0.001$ \\
\hline Mean total charges (SD), in USD & \multicolumn{2}{|c|}{$207,765(118,932)$} & \multicolumn{2}{|c|}{$223,568(130,000)$} & $<0.001$ \\
\hline In-hospital mortality & 780 & 3.6 & 605 & 5.1 & $<0.001$ \\
\hline
\end{tabular}

TABLE 1: Differences in demographics and inpatient outcomes in aortic stenosis

SD: standard deviation; USD: United States dollars

Female patients with AS have 1.35 times higher odds (95\% CI 1.20-1.51) for in-hospital mortality compared to males. When compared to whites, no statistically significant association with inpatient mortality was seen in any other race. Among comorbidities, the association with in-patient mortality was seen with coagulopathy by two times ( $95 \%$ CI 1.79-2.27), and CHF by 1.62 times (95\% CI 1.39-1.89). After controlling for demographic confounders and other comorbidities, the odds of in-hospital mortality was higher in patients with comorbid AF by 1.43 times (95\% CI 1.28-1.61) as shown in Table 2. 


\section{Cureus}

\begin{tabular}{|c|c|c|c|c|}
\hline \multirow{2}{*}{ Variables } & \multirow{2}{*}{ Odds ratio } & \multicolumn{2}{|c|}{ 95\% Confidence interval } & \multirow{2}{*}{ P-value } \\
\hline & & Lower & Upper & \\
\hline Age & 1.02 & 1.01 & 1.03 & $<0.001$ \\
\hline \multicolumn{5}{|l|}{ Sex } \\
\hline Male & Reference & & & \\
\hline Female & 1.35 & 1.20 & 1.51 & $<0.001$ \\
\hline \multicolumn{5}{|l|}{ Race } \\
\hline White & Reference & & & \\
\hline Black & 0.86 & 0.63 & 1.17 & 0.332 \\
\hline Hispanic & 0.92 & 0.65 & 1.29 & 0.614 \\
\hline Other & 1.08 & 0.83 & 1.39 & 0.574 \\
\hline \multicolumn{5}{|l|}{ Comorbidities } \\
\hline No comorbidities & Reference & & & \\
\hline Chronic pulmonary disease & 1.09 & 0.98 & 1.24 & 0.123 \\
\hline Congestive heart failure & 1.62 & 1.39 & 1.89 & $<0.001$ \\
\hline Coagulopathy & 2.02 & 1.79 & 2.27 & $<0.001$ \\
\hline Renal failure & 1.43 & 1.28 & 1.61 & $<0.001$ \\
\hline
\end{tabular}

TABLE 2: Risk of in-hospital mortality in aortic stenosis

\section{Discussion}

Our study used the inpatient data from the US hospitals and found that the prevalence of RF in AS patients is $29.2 \%$, and a higher proportion these patients were male (60.1\%) and non-white being black, Hispanic, or other race (14.1\%) compared to the non-RD cohort. Female patients had higher odds of in-hospital mortality in AS patients. Race was a non-significant predictor for mortality risk in our study inpatients. Our study showed that AS patients with comorbid coagulopathy and CHF had increased mortality. Major loss of function, severity of illness, and in-hospital mortality were seen in a higher proportion of AS inpatients with comorbid RF compared to the non-RF cohort.

AS is a common valvular abnormality with an approximate overall prevalence of $3 \%$ in the elderly over 75 years in the western world [10]. The incidence of AS rises with age, affecting up to $10 \%$ of the elderly by the eighth decade [11]. Risk factors associated with AS are older age, comorbidities such as diabetes and chronic renal failure, history of prior heart failure and left ventricular dysfunction, cardiac ischemic event, or revascularization [2]. When aortic valve sclerosis (thickening and calcification of the aortic valve) affects about $25 \%$ of elderly people then AS is prevalent in $2 \%$ to $9 \%$ of the elderly population over 65 years of age. A rise in prevalence was seen for both aortic sclerosis (48\%) and AS (4\%) in the elderly aged above 85 years [2].

We found that AS patients with comorbid RF had a higher prevalence of comorbidities (90.5\%), with a higher proportion of coagulopathy $(27.4 \%)$ and CHF (12.7\%). One of the studies showed that most patients with ESRD have numerous comorbidities along with experiencing effects of advanced AS and prognosis of ESRD patients is highly affected by the presence of other comorbidities and their functional status. Comorbidities like peripheral vascular disease, coronary artery disease, CHF, and diabetes are associated with nearly $50 \%$ one-year survival in elderly patients on dialysis [4]. Another meta-analysis suggested that the occurrence of $\mathrm{RF}$ requiring hemodialysis following TAVR is related to baseline comorbidities instead of the amount of contrast used [12]. The presence of valvular abnormality is associated with higher in-hospital and 30-day mortality, and the clinical factors related to AS and aortic sclerosis are alike the risk factors for atherosclerosis, that is, old age, male gender, smoking, hypertension, and dyslipidemia [2].

As per our study, there was a statistically significant difference with higher mean LOS in AS inpatients with comorbid RF. According to a study, AKI accounts for up to a four-fold rise in one-year mortality, and individuals who developed AKI after TAVR had a mortality rate of $7.8 \%$ to $29 \%$ which was two to eight times 
higher as compared to patients who did not suffer from AKI. Also, AKI was associated with a higher LOS in AS patients who underwent TAVR, with a rise of about 2.5 times [6]. Renal dysfunction is one of the major factors responsible for adverse outcomes in individuals undergoing TAVR. Individuals with renal dysfunction usually have a high number of comorbidities and that may severely affect their life after TAVR.

The existence of renal dysfunction also affects the natural course of AS, probably via a mechanism that further contributes to calcium deposition in aortic leaflets, thus deteriorating AS. Advanced AS with reduced blood supply to major organs also contributes to the onset of complications, therefore enhancing the mortality risk after TAVR [12].

Deteriorating renal function manifested by estimated glomerular filtration rate (eGFR) decline was independently linked with a rise in all-cause mortality in high-risk individuals with AS. Initial stages of both chronic kidney disease and ESRD are associated with enhanced coronary as well as aortic and mitral valvular calcification and thus, a rise in parathyroid hormone, calcium-phosphate products, and excess 1,25 hydroxyvitamin D among other metabolic abnormalities. Valvular calcification is also associated with an enhanced rate of progression of AS and poor outcomes [13]. Increased morbidity and mortality related to TAVR are likely secondary to heavy calcification in the vasculature, aorta, and aortic annulus leading to bleeding and vascular complications [7].

A study showed that there are a variety of factors linked with AKI following TAVR such as high preoperative creatinine levels, the need for blood transfusion, peripheral vascular disease, and hypertension and thus, the short term mortality (<30 days) in patients who developed AKI after TAVR and was higher compared to those without AKI [14]. Baseline glomerular filtration rate is an independent predictor of acute kidney injury in patients undergoing TAVR which adversely affects 30-day and one-year mortality [15]. A study found that male sex, left ventricular ejection fraction $<30 \%$, atrial fibrillation, and advanced chronic kidney disease were independently associated with higher mortality in patients receiving TAVR in predialysis or dialysis individuals [16]. The presence of both severe chronic kidney disease and reduced LVEF and/or deteriorating functional status has a gradually progressive effect on mortality risk after TAVR [17]. Chronic kidney disease is predictive of enhanced 30-day and one-year all-cause mortality, AKI, dialysis requirement, adverse short and long-term outcomes following TAVR [18]. According to a multi-center study, individuals with advanced chronic kidney disease have remarkably high long-term mortality rates and also, various other factors such as age, male sex, lower ejection fraction are independently linked with high mortality rates following TAVR [16].

After controlling confounders, we found that comorbid RF was significantly associated with higher inhospital mortality in AS inpatients in the post-TAVR period. According to a study, individuals with ESRD have increased in-hospital mortality, increased occurrence of in-hospital complications, and poor outcomes following TAVR. AKI is linked with increased in-hospital mortality in patients undergoing TAVR [7]. Individuals with advanced AS with ESRD who undergo TAVR have a remarkably elevated risk of mortality at 30 days and one year, and have increased in-hospital mortality and bleeding as compared to non-dialysis dependent individuals [4]. A decrease in eGFR in individuals with chronic kidney disease and AS is associated with an $18 \%$ higher risk of all-cause mortality [13]. A meta-analysis demonstrated that females who underwent TAVR had greater long-term survival as compared to males but an increased short-term risk ( $<30$ days) of major bleeding, vascular complications, and stroke [19].

There are a few limitations to our study. Administrative data from NIS were used to study inpatients as per the ICD-9 codes which may cause underreporting of comorbidities, including RF, thus affecting the statistical analyses. Since it is an observational cross-sectional study and all-cause in-hospital mortality was included, a causal relationship can't be proved between mortality and comorbidities in AS. In order to strengthen our study, we utilized the NIS data covering hospitals from 44 states in the United States and the results have appropriate generalizability for the inpatient population. A logistic regression model adjusted for demographic confounders and comorbidities was used and thus, we evaluated the association of comorbidities including comorbid RF with in-hospital mortality in AS inpatients undergoing TAVR.

\section{Conclusions}

Renal failure was prevalent in AS patients and was an independent factor that increases the risk of inhospital mortality by $43 \%$. Due to worse outcomes, more studies are required to evaluate the risk-benefit ratio and strategies to improve health-related quality of life in post-TAVR patients with RF, and optimally decrease inpatient mortality. Periodic renal function tests and appropriate management of chronic kidney disease need to be implemented at initial stages to improve health-related quality of life and decrease inhospital mortality in AS patients.

\section{Additional Information}

\section{Disclosures}

Human subjects: Consent was obtained by all participants in this study. Animal subjects: All authors have confirmed that this study did not involve animal subjects or tissue. Conflicts of interest: In compliance 
with the ICMJE uniform disclosure form, all authors declare the following: Payment/services info: All authors have declared that no financial support was received from any organization for the submitted work. Financial relationships: All authors have declared that they have no financial relationships at present or within the previous three years with any organizations that might have an interest in the submitted work. Other relationships: All authors have declared that there are no other relationships or activities that could appear to have influenced the submitted work.

\section{References}

1. Lindman BR, Clavel MA, Mathieu P, Iung B, Lancellotti P, Otto CM, Pibarot P: Calcific aortic stenosis. Nat Rev Dis Primers. 2016, 2:16006. 10.1038/nrdp.2016.6

2. Faggiano P, Antonini-Canterin F, Baldessin F, Lorusso R, D'Aloia A, Cas LD: Epidemiology and cardiovascular risk factors of aortic stenosis. Cardiovasc Ultrasound. 2006, 4:27. 10.1186/1476-7120-4-27

3. Saran R, Robinson B, Abbott KC, et al.: US renal data system 2017 annual data report: epidemiology of kidney disease in the united states. Am J Kidney Dis. 2018, 71:7. 10.1053/j.ajkd.2018.01.002

4. Szerlip M, Zajarias A, Vemalapalli S, et al.: Transcatheter aortic valve replacement in patients with endstage renal disease. J Am Coll Cardiol. 2019, 73:2806-2815. 10.1016/j.jacc.2019.03.496

5. Thourani VH, Forcillo J, Beohar N, et al.: Impact of preoperative chronic kidney disease in 2,531 high-risk and inoperable patients undergoing transcatheter aortic valve replacement in the partner trial. Ann Thorac Surg. 2016, 102:1172-1180. 10.1016/j.athoracsur.2016.07.001

6. Najjar M, Salna M, George I: Acute kidney injury after aortic valve replacement: incidence, risk factors and outcomes. Expert Rev Cardiovasc Ther. 2015, 13:301-316. 10.1586/14779072.2015.1002467

7. Gupta T, Goel K, Kolte D, et al.: Association of chronic kidney disease with in-hospital outcomes of transcatheter aortic valve replacement. JACC Cardiovasc Interv. 2017, 10:2050-2060. 10.1016/j.jcin.2017.07.044

8. Overview of the national (nationwide) inpatient sample . (2019). Accessed: March 15, 2020: https://www.hcup-us.ahrq.gov/nisoverview.jsp.

9. NIS description of data elements. (2019). Accessed: March 15, 2020: https://www.hcupus.ahrq.gov/db/nation/nis/nisdde.jsp.

10. Carita P, Coppola G, Novo G, et al.: Aortic stenosis: insights on pathogenesis and clinical implications . J Geriatr Cardiol. 2016, 13:489-498. 10.11909/j.issn.1671-5411.2016.06.001

11. Joseph J, Naqvi SY, Giri J, Goldberg S: Aortic stenosis: pathophysiology, diagnosis, and therapy. Am J Med. 2017, 130:253-263. 10.1016/j.amjmed.2016.10.005

12. Chen C, Zhao ZG, Liao YB, et al.: Impact of renal dysfunction on mid-term outcome after transcatheter aortic valve implantation: a systematic review and meta-analysis. PLoS One. 2015, 10:e0119817. 10.1371/journal.pone.0119817

13. Patel KK, Shah SY, Arrigain S, et al.: Characteristics and outcomes of patients with aortic stenosis and chronic kidney disease. J Am Heart Assoc. 2019, 8:009980. 10.1161/JAHA.118.009980

14. Elhmidi Y, Bleiziffer S, Deutsch MA, Krane M, Mazzitelli D, Lange R, Piazza N: Acute kidney injury after transcatheter aortic valve implantation: incidence, predictors and impact on mortality. Arch Cardiovasc Dis. 2014, 107:133-139. 10.1016/i.acvd.2014.01.002

15. Meneguz-Moreno RA, Ramos AI, Siqueira D, et al.: Prognostic value of renal function in patients with aortic stenosis treated with transcatheter aortic valve replacement. Catheter Cardiovasc Interv. 2017, 89:452-459. 10.1002/ccd.26693

16. Conrotto F, Salizzoni S, Andreis A, et al.: Transcatheter aortic valve implantation in patients with advanced chronic kidney disease. Am J Cardiol. 2017, 119:1438-1442. 10.1016/j.amjcard.2017.01.042

17. Levi A, Codner P, Masalha A, et al.: Predictors of 1-year mortality after transcatheter aortic valve implantation in patients with and without advanced chronic kidney disease. Am J Cardiol. 2017, 120:20252030. 10.1016/j.amjcard.2017.08.020

18. Ifedili IA, Bolorunduro O, Bob-Manuel T, Heckle MR, Christian E, Kar S, Ibebuogu UN: Impact of preexisting kidney dysfunction on outcomes following transcatheter aortic valve replacement. Curr Cardiol Rev. 2017, 13:283-292. 10.2174/1573403X13666170804151608

19. Saad M, Nairooz R, Pothineni NVK, et al.: Long-term outcomes with transcatheter aortic valve replacement in women compared with men: evidence from a meta-analysis. JACC Cardiovasc Interv. 2018, 11:24-35. 10.1016/j.jcin.2017.08.015 